\title{
Percepções dos praticantes de Crossfit: um estudo exploratório
}

Crossfit participant's perceptions: an exploratory study

Percepciones de los practicantes de Crossfit: un estudio exploratorio

Fernanda Celeste da SILVA

Bruno Eduardo ALVES

Lucas Mendes MORAES

Rafael Luiz Del Buono NEMEZIO

Daniel Zonzini VOLTAN

Centro Universitário de Jaguariúna - UNIFAJ, 13820-000 Jaguariúna - SP, Brasil

\section{Resumo}

O Crossfit tem se firmado ao redor do mundo com forte apelo mercadológico e tem atraído milhões de pessoas para seu box. Com mais de 10 mil academias licenciadas, é inegável este fenômeno da prática corporal mundial. Quanto ao campo da ciência, os estudos caminham na direção da eficácia do treinamento e lesões. Porém pouco se sabe sobre o que os participantes pensam sobre o método em caráter científico. Assim, o objetivo deste estudo foi analisar o grau de conhecimento e percepções dos praticantes do método Crossfit. Realizou-se a aplicação de questionário em 270 praticantes do método em quatro cidades diferentes onde foi possível observar que a maioria: acredita que conhece a modalidade mesmo tendo algumas respostas conflitantes; relaciona Crossfit com saúde e busca o mesmo com este objetivo; praticam somente a modalidade e não tiveram lesões advindas da prática; acham o ambiente motivador e não possuem interesse em competir; que o conhecimento técnico é a característica mais importante em um coach e que seu próprio coach conhece muito sobre a modalidade. Assim, novos estudos devem ser considerados sobre a modalidade tanto do ponto de vista do treinamento quanto da percepção dos seus praticantes, pois muitos indivíduos não têm o conhecimento mínimo sobre este treinamento e acabam tendo uma visão distorcida ou muito vaga sobre o assunto.

Descritores: Educação Física e Treinamento; Academias de Ginástica; Percepção Social.

\section{Abstract}

Crossfit has established around the world with strong marketing appeal and has attracted millions to its box. With more than 10,000 licensed academies, this body moviment practice phenomenon is undeniable. In the field of science, studies are concerned to training effectiveness and injury. But there is not to many works about what the participants think about the method in a scientific way. Thus, the objective of this study was to analyze the degree of knowledge and perceptions of participants of the Crossfit method. A questionnaire was applied to 270 participants of the method in four different cities where it was possible to observe that the majority: believes that they know the modality even though they have some conflicting answers; relates Crossfit to health and pursues this as a goal; they only practice the modality and did not have injuries resulting from the practice; they find the environment motivating and have no interest in competing; that technical knowledge is the most important characteristic in a coach and that his own coach knows a lot about the Crossfit. Thus, new studies should be considered about the modality both from the training point of view and the perception of its practitioners, since many individuals do not have the minimum knowledge about this training and end up having a distorted or very vague view on the subject.

Descriptors: Physical Education and Training; Fitness Centers; Social Perception.

\section{Resumen}

Crossfit se ha establecido en todo el mundo con un fuerte atractivo de marketing y ha atraído a millones de personas a su box. Con más de 10,000 academias autorizadas, este fenómeno de práctica corporal mundial es innegable. En cuanto al campo de la ciencia, los estudios avanzan hacia la efectividad del entrenamiento y las lesiones. Sin embargo, se sabe poco sobre lo que los participantes piensan sobre el método de manera científica. Por lo tanto, el objetivo de este estudio fue analizar el grado de conocimiento y las percepciones de los profesionales del método Crossfit. Se aplicó un cuestionario a 270 practicantes del método en cuatro ciudades diferentes donde fue posible observar que la mayoría: creen que conocen el deporte incluso con algunas respuestas conflictivas; relaciona Crossfit con la salud y busca lo mismo para este propósito; ellos solo practican el deporte y no tuvieron lesiones de la práctica; encuentra el ambiente motivador y no tiene interés en competir; que el conocimiento técnico es la característica más importante de un entrenador y que su propio entrenador sabe mucho sobre el deporte. Por lo tanto, se deben considerar nuevos estudios sobre la modalidad, tanto desde el punto de vista del entrenamiento como de la percepción de sus profesionales, ya que muchas personas no tienen el mínimo conocimiento sobre este entrenamiento y terminan teniendo una visión distorsionada o muy vaga sobre el tema.

Descriptores: Educación y Entrenamiento Físico; Centros de Acondicionamiento; Percepción Social.

INTRODUÇÃO

O método de treinamento Crossfit vem se tornando a modalidade mais procurada entre os indivíduos que procuram programas de treinamento mais intenso e desafiadores. $O$ Crossfit teve sua origem em meados da década de 90, na Califórnia, quando um ex-ginasta chamado Greg Glassaman começou a desenvolver um novo método de treinamento composto por exercícios funcionais, de alta intensidade e com foco em desenvolver o condicionamento físico de uma forma geral, sem focar apenas na especialização de uma determinada habilidade, como acontece nos treinos tradicionais ${ }^{1,2}$. Atualmente existem mais de 10 mil academias licenciadas pela Crossfit
Inc®. em mais de 97 países, além de existir academias que utilizam a metodologia do Crossfit, mas não faz uso da marca. O Crossfit é um método de treinamento novo caracterizado pela realização de exercícios funcionais, exercícios básicos e exercícios de potência, constantemente variados em alta intensidade ${ }^{2,4}$. O programa se destaca pela forte divulgação midiática e pelo fator de ser um treinamento competitivo e motivador. Fundado sobre três pilares (intensidade, variação e funcionalidade), o Crossfit tem por objetivo melhorar o condicionamento físico de forma global, preparando o indivíduo para as mais variadas tarefas físicas ${ }^{2}$. Segundo seu criador, o Crossfit 
é supostamente superior a outras modalidades, pelo fato de se treinar diversas capacidades físicas de maneira simultânea ${ }^{1,2}$. Porém, é difícil saber ainda como o uso do método implica nas questões de segurança e eficácia, nesta direção vários estudos tem se debruçado sobre esta temática com o intuito de esclarecer e fortalecer a modalidade, tanto daqueles que conhecem a modalidade como qualquer outra pessoa leiga no assunto ${ }^{4,5}$.

O foco nas publicações acerca do Crossfit tem se voltado para as ações do $\operatorname{coach}^{1,6,7}$, aspectos nutricionais ${ }^{8-10}$, e aspectos psicológicos ${ }^{11-13}$ como motivação, engajamento e senso de comunidade. Com mais impacto estão os trabalhos sobre traumas ${ }^{14}$, lesões ${ }^{15,16}$, como as osteo-articulares ${ }^{17-20}$, as tendíneas ${ }^{21}$ e musculares $^{22}$ e rabdomiólise ${ }^{23-28}$. Entretanto, o maior esforço de pesquisa está voltado sobre o treinamento da modalidade do ponto de vista biomecânico $^{14}$, fisiológico ${ }^{29-34}$ e intervenções práticas $^{35,36}$, inclusive 0 uso do programa em militares americanos ${ }^{37-40}$ e para adolescentes ${ }^{41}$.

Em estudo realizado acerca do Crossfit, encontrou-se 31 artigos para revisão sistemática e 4 para meta-análise. Tais artigos investigaram os efeitos do Crossfit na composição corporal, parâmetros psicofisiológicos, risco de lesão musculoesquelética, aspectos de vida e saúde e comportamento psicossocial. A meta-análise não encontrou um efeito significativo de mudanças no treinamento Crossfit no índice de massa corporal, gordura corporal relativa, massa gorda, massa magra e circunferência da cintura $^{42}$.

Entretanto, pouco se sabe o que o praticante entende acerca da modalidade Crossfit. As intenções da prática esportiva sugerem o esporte-educação, o esporte-lazer e o esporte-competição ${ }^{43}$. O Crossfit entendido aqui como prática corporal também pode ser compreendido sob os dois últimos contextos. Assim, a pergunta norteadora deste trabalho: Em que grau o praticante de Crossfit conhece a modalidade e como se estrutura sua autopercepção?

Dessa forma, justifica-se então a apresentação de um novo ponto de vista sobre a modalidade, tal qual 0 entendimento, 0 conhecimento e percepção da prática sob a ótica do praticante. Não é premissa deste trabalho por fim à esta discussão, mas sim trazer à tona como este indivíduo pensa a modalidade e interage com ela. Então, o presente estudo não busca resposta em relação ao treinamento em si, mas tem o objetivo de analisar o grau de conhecimento e percepções dos praticantes do método Crossfit.
MATERIAL E MÉTODO

Trata-se de um estudo exploratório em que os indivíduos responderam à um formulário escrito de perguntas fechadas sem a necessidade de identificação ${ }^{44,45}$. Durante 0 preenchimento os pesquisadores podiam auxiliar, caso fosse solicitado pelos indivíduos pesquisados.

A coleta ocorreu no ano de 2017 e envolveu academias que utilizam o treinamento baseado na modalidade Crossfit sem a necessidade de estarem filiadas à marca, nas cidades brasileiras de Amparo-SP, Artur Nogueira-SP, Mogi Mirim-SP e Mogi Guaçu-SP que autorizaram previamente a realização da pesquisa.

A amostra foi determinada por conveniência e foi composta de 270 indivíduos sendo 125 homens e 145 mulheres. Os critérios para a inclusão neste estudo foram todos os indivíduos, de ambos os sexos, matriculados na modalidade Crossfit nos locais de treinamento, independente do tempo de prática e que concordaram em participar autorizando o uso das respostas através da assinatura do TCLE. Foram excluídos da pesquisa todos aqueles menores de 18 anos de idade. Esta pesquisa foi aprovada pelo Comitê de Ética em Pesquisa sob Parecer no: 2.003.086.

Realizou-se uma análise estatística descritiva simples para encontrar a aglutinação de opiniões dos participantes. Para isso, foi utilizado o programa Excel® 2017 da Microsoft Office $\AA^{\circ}$.

RESULTADOS E DISCUSSÃO

A Tabela 1 apresenta os dados obtidos na pesquisa de campo, representados em porcentagem de acordo com questionário aplicado.

Ficou evidente que a maioria das pessoas, $48 \%$, que praticam Crossfit acreditam que possuem um conhecimento razoável da modalidade. Isso não significa que de fato conheçam a modalidade com profundidade. É bem provável que o conhecimento destes indivíduos venha de sites e blogs na internet sem qualquer tratamento acadêmico ou científico. E notável que uma parte bem significante dos indivíduos, 35\%, conheça pouco sobre o treinamento. Estas informações fazemse parecer óbvio que algumas pessoas vão à procura do Crossfit sem ao menos saber o que realmente ele oferece e como as aulas ocorrem. Ainda, ao que parece, os $11 \%$ que conhecem muito pouco sobre a modalidade devem ter ingressado pelo momento midiático em que a mesma se encontra ou por indicação de amigos. 
Tabela 1. Dados obtidos após a aplicação do questionário 1. Quanto você acha que conhece sobre a modalidade Crossfit? Conheço muito pouco

Conheço pouco

Conheço bastante

2. Você pratica outras atividades além do Crossfit?

Somente Crossfit
Pratico mais 1 ou 2 modalidades

3. Qual é o seu objetivo com a prática do Crossfit?

Competição

Relacionamento social

Lazer

4. Você relacionaria a modalidade Crossfit com saúde?

\begin{tabular}{l} 
4. Voce \\
Sim \\
\hline Não
\end{tabular}

Não

5. Você já teve alguma lesão, devido a prática do Crossfit?

Sim

6. Assinale as opcĩes abaixo que mais representam o ambiente do Box que freque

(a)

Competitivo

Desafiador

Desatiador

Acolhedor

7. Tem interesse em competir na modalidade Crossfit?

Sim

Não

8. Assinale 2 características abaixo que você considera importantes no SEU Coach: Conhecimento técnico

Didática

Motivação

9. Quanto você acha que o seu Coach conhece sobre a modalidade Crossfit?

Conhece pouco

Tem um conhecimento razoáv

Conhece bastante

10. Qual a probabilidade de você indicar o Crossfit para um familiar ou amigo?

Não indicaria

Indicaria com ressalvas

Quanto a realizar outras atividades, aproximadamente $59 \%$ dos indivíduos que foram avaliados praticam somente o Crossfit. Isso pode sugerir que estes indivíduos podem se dedicar totalmente à modalidade. Do ponto de vista da prescrição do exercício físico, não haveria grandes problemas para ajustar os volumes e intensidades do treino. De forma inversa, os entrevistados revelam que não conhecem muito a respeito da modalidade que pratica, sendo assim, difícil estipular metas e traçar objetivos a serem conquistados. Não se pode esquecer dos $41 \%$ que praticam outras modalidades e, isto pode gerar algum conflito nos resultados propostos pela prática, sendo difícil de atribuir exclusivamente ao Crossfit a melhora no condicionamento físico como dito anteriormente ${ }^{2}$.

Quando perguntado sobre os objetivos da prática do Crossfit, os indivíduos tinham a opção de preencher quantos objetivos eles julgassem cabíveis. Observa-se que metade das respostas, $55 \%$, foram assinaladas como objetivo principal a busca por saúde. Tal resultado pode ser reflexo da vasta exposição midiática e médica dos fortemente estabelecidos estudos que apresentam 0 exercício físico como um dos principais meios de melhoria e manutenção dos índices de saúde.

De qualquer maneira, às custas da saúde muitas vezes, $20 \%$ das respostas foram na direção da estética. Sendo a beleza o ponto mais alto de uma hierarquia de valores, onde a ascensão social, profissional e afetiva são encaradas como uma competição que finda somente em atributos físicos ${ }^{46,47}$. Vale ressaltar que, muitas vezes, a busca pela estética pode ultrapassar o bom senso da prática saudável, podendo provocar lesões ou ainda outros problemas em longo prazo.

Ainda, $13 \%$ assinalaram a prática por lazer, de caráter "desinteressado" da vivência sendo realizada no tempo de livre de todas as obrigações profissionais, sociais e familiares ${ }^{48,49}$. Em tempo, $7 \%$ procuram por competição e $5 \%$ por relacionamento pessoal, sendo a academia para este último um excelente espaço para esta convivência ${ }^{50}$.

Isso nos leva a considerar todos os indivíduos e suas intenções de prática. Aqueles que praticam não entendem que modalidades altamente competitivas não trazem grandes benefícios à saúde devido às cargas elevadas de treinos e periodizações rigorosas com objetivos específicos. Muitas vezes o mesmo indivíduo assinalou saúde e competição, ou ainda, saúde, estética e lazer. Sendo esses, objetivos dicotômicos muitas vezes. Exemplo disso é a questão do lazer, sendo este um conceito de difícil entendimento pelos praticantes. Pois, quando estes vão para o treinamento, mesmo que seja no seu tempo livre de obrigações, o treino não tem o fim em si mesmo comprometendo assim o conceito in natura do lazer.

Um estudo analisou as possíveis motivações que levaram os sujeitos à procurarem 0 Crossfit aplicando 566 questionários e as respostas mais assinaladas foram melhorar condicionamento físico, melhorar qualidade de vida, curiosidade sobre o método e estética ${ }^{19}$. Comparando estes resultados com o presente estudo é possível traçar um paralelo entre qualidade de vida, saúde e condicionamento físico.

Ao que parece, os objetivos não são bem definidos pelo coach junto dos alunos quando estes ingressam nesta modalidade de treinamento. Coach é a maneira como é chamado o "Treinador" na modalidade, independente da formação acadêmica. O coach no Crossfit é aquele que possui as certificações específicas para ensinar e treinar a partir do método exclusivo do Crossfit. Por outro lado, não significa que as interações sociais não ocorrerão por questões de objetivos diferentes. O que se deve ter em mente é a montagem das turmas e dos treinos de acordo com os objetivos de treinamento.

Acerca da relação do Crossfit e saúde, 
praticamente todos os indivíduos acreditam que eles estejam interligados. Mesmo levando em consideração a subjetividade, a dinâmica, cultura e os aspectos físicos e mentais do conceito de saúde ${ }^{51,52}$ seria um erro assumir com segurança esta relação. Para o criador da modalidade o condicionamento físico e a saúde são a mesma coisa ${ }^{53}$. E este é um conceito que não se encontra alinhado com a literatura especializada recorrente, pois sabe-se que este estado de saúde é multifatorial e interdependente, não podendo assim, concordar que condicionamento físico seja a mesma coisa que saúde.

Sabe-se que o Crossfit, assim como qualquer outro treinamento de alta intensidade, aumenta $\mathrm{VO}_{2 \text { MAX }}$, força, hipertrofia e resistência, e diminui a massa magra ${ }^{15,54,55}$. E, como visto anteriormente, o direcionamento da pesquisa científica no Crossfit está primordialmente relacionada ao seu treinamento e lesões.

Porém, no treinamento da modalidade Crossfit a prescrição não é individualizada e os princípios da Individualidade, Adaptação e da Sobrecarga não são a base da escolha dos exercícios e da progressão de carga. A Individualidade biológica, que explica a variabilidade entre elementos da mesma espécie $^{56}$, apresenta-se como mudanças funcionais e estruturais em quase todos os sistemas a partir das atividades psicofísicas e esportivas ${ }^{57}$ podendo ser considerada um dos mais importantes princípios do treinamento. Já o princípio da Especificidade ${ }^{58}$ é contemplado pelo fato da modalidade possuir características específicas. Há que se verificar os critérios dos princípios da Continuidade uma vez que vários praticantes não assumem a modalidade enquanto competição profissional. O caso do princípio da Interdependência VolumeIntensidade talvez seja o mais interessante e perigoso. Pois, na prescrição do Crossfit, o movimento é obrigatoriamente variado e de alta intensidade ${ }^{53}$.

Sendo assim, a busca é constante pela máxima carga e máximo volume possível. Aliás, característica marcante da modalidade quando praticada no formato competitivo. No que tange à prescrição segura, um fator é alarmante é o fato da metodologia por trás do Crossfit ser completamente empírica ${ }^{53}$. Quando relacionado com qualidade de vida, o Crossfit, obviamente proporciona adaptações morfofisiológicas com consequente melhora na capacidade física ${ }^{59}$, o que pode promover qualidade de vida aos praticantes. Porém, é preciso ter cautela na generalização desta afirmação porque 0 processo de treino de maneira igualitária pode não trazer os mesmos benefícios para todos os praticantes. Além do fato de qualidade de vida ser um conceito subjetivo ${ }^{60-62}$.

Sobre o Crossfit e lesões, os resultados mostraram que $88 \%$ dos indivíduos não se lesionaram com a prática. Entretanto, não é possível negligenciar os lesionados. Mesmo que esta modalidade tenha um forte apelo mercadológico, é importante ter cautela na generalização de sua aplicação. Embora as evidências científicas existentes sobre o programa ainda sejam controversas, elas apontam elevado risco de lesões ao Crossfit ${ }^{63}$

Estas lesões poderiam estar associadas à execução errada do movimento, ou excesso de cargas, ou ainda, lesões musculares causadas por fadiga. De qualquer maneira, em estudo com 980 indivíduos que treinam Crossfit encontraram taxas de lesões no ombro durante o treinamento Crossfit são comparáveis aos outros métodos de exercício recreativo ${ }^{18}$. Outro artigo que vem corroborar com os apresentados aqui, é uma revisão sistematizada que afirma que o Crossfit é comparável a outros métodos de treinamento de alta intensidade em termos de taxas de lesão e saúde. Sobre os fatores que podem apresentar maiores probabilidades de ter lesões musculoesqueléticas estão o sobrepeso e obesidade, fazer outra atividade física além do Crossfit, praticar mais de três vezes por semana com um tempo diário acima de uma hora de treino, fazer uso de suplementos alimentares, ser sexo masculino, fazer uso de bebidas alcoólicas e cigarro ${ }^{59}$.

Temática sempre presente entre os praticantes da modalidade está a rabdomiólise entendida como o conjunto de sintomas clínicos e achados laboratoriais consequentes do extravasamento de conteúdos intracelulares de miócitos para a corrente sanguínea, em especial eletrólitos, mioglobina e proteínas sarcoplasmáticas ${ }^{64}$. Tal decorrência não parece ter fortes evidências de correlação, porém sugere-se cautela na prescrição para evitar tal condição gerada pela má prescrição.

Para garantir a eficácia do método o site oficial da marca expressa que a escala dos exercícios deve ser direcionada às necessidades exclusivas de cada atleta adequadamente $^{65}$. Ora um tanto quanto contraditória uma vez que a prescrição é a mesma para todos no Crossfit.

Outro ponto que merece consideração é o fato dos estudos, assim como o este, analisar somente os indivíduos que continuam praticando a modalidade. Precisa-se entender que, provavelmente outros indivíduos já se lesionaram e não praticam mais a modalidade, 
formando assim um grupo importante a se pesquisado. Em tempo, os desenhos metodológicos para garantir a qualidade das evidências ainda está em construção e não apresenta uma constância de dados, ou ainda são tendenciosos. Box é o nome atribuído ao local onde ocorre a prática do método Crossfit. Dentro deste aspecto, para $46 \%$ dos entrevistados este ambiente mostra-se motivador. A característica desafiadora aparece em segundo lugar com $27 \%$. E em terceiro com $23 \%$, o ambiente aparenta ser acolhedor. Isso nos leva a crer que o local em que os indivíduos praticam a modalidade garantem sua permanência no treinamento a partir das relações que lá acontecem, como motivação entre coach-alunos e alunos-alunos. Mesmo que haja uma certa dificuldade em todos os integrantes executarem 0 wod, 0 ambiente parece ser muito solidário a todos que tentam enfrentar as prescrições diárias propostas. Um estudo pioneiro ${ }^{13}$ medindo o "senso de comunidade" entre praticantes de uma academia de ginástica tradicional e uma no modelo de academia Crossfit usando análise adaptada do Capital Social e Escalas Gerais de Pertencimento como ferramentas metodológicas para comparar as percepções entre as duas academias. Os membros do Crossfit relataram níveis significativamente mais altos de capital social e pertencimento à comunidade em comparação com os membros tradicionais da academia. Entretanto, o estudo não mostrou que 0 capital social, 0 pertencimento à comunidade e nem o tipo de academia eram um preditor independente da manutenção da frequência de treino. Outros pesquisadores também relatam que os participantes da Crossfit foram mais propensos a reportar níveis mais altos de motivos intrínsecos, como gozo, desafio e afiliação, ao passo que os clientes de personal trainning relataram valores mais elevados por motivos relacionados à saúde, tais como saúde positiva, prevenção de doenças e gerenciamento de peso ${ }^{11}$.

Mesmo que o ambiente do treinamento seja motivador e desafiador, grande parte dos indivíduos não tem interesse na competição, característica essa que originou a modalidade. Pouco mais de $1 / 3$ possui interesse em competição. É possível observar que vários termos não estão bem claros para os praticantes, onde $36 \%$ tem a vontade de competir. Entretanto, mais de $50 \%$ dos entrevistados buscam por saúde, sendo que estas são duas metas totalmente contrárias. Embora o ambiente proporcione uma certa competitividade entre os praticantes, $20 \%$ não se mostraram decididos quanto aos objetivos específicos do Crossfit.

Sobre o que os indivíduos da pesquisa pensam sobre o seus coaches, o conhecimento técnico e como a característica o mais importante. Uma vez que, o Crossfit busca promover técnicas de treinamento para 0 público em geral ${ }^{53}$, fica clara a importância da formação contínua do profissional de Educação Física no que tange aos aspectos técnicos e pedagógicos na relação aluno/professor. No Brasil, existe a necessidade de formação acadêmica em Educação Física para ser um coach.

Outro estudo mostrou que aplicar o método com conhecimento superficial pode trazer sérios danos à saúde do aluno e mercadologicamente afeta de forma negativa a modalidade podendo ser constatado quando as taxas de lesões foram diretamente relacionadas à supervisão dos treinadores da Crossfit. Vários são os fatores que influenciam neste processo, como por exemplo a organização da classe e correção da técnica, ajuste da carga correta para o treino. $\mathrm{Na}$ maioria das vezes, os exercícios são prescritos com o volume e intensidade para toda a classe, o que pode obviamente será excessivo para membros menos experientes. Outro fator apontado foi que o nível de experiência do atleta e o envolvimento geral dos treinadores teve alta correlação com a saúde dos atletas ${ }^{66}$.

Em entrevista com 6 donos/coaches de academias de Crossfit foram revelados os seguintes fatores-chaves que podem tanto facilitar quanto restringir a prática da modalidade a partir do ponto de vista dos coaches: consideração administrativa; inclusão, tolerância e empoderamento; oportunidade de liderança; interesses em comum; competitividade; suporte social; espaços sociais; e ações voluntárias. Trazem ainda: sugestões sobre como melhorar a iniciação na modalidade e sua adesão; estratégias para 0 desenvolvimento da comunidade; medidas para reduzir intimidação e custo; e facilitar metas realistas e garantir 0 apoio social. Todo este processo mapeado pelos autores levam à criação do que eles denominam 'senso de comunidade' entre os praticantes ${ }^{6}$.

Para maioria esmagadora dos indivíduos responderam que seus coaches conhecem bastante do programa de treinamento que estão aplicando. É recomendado que os participantes avaliem a habilidade e a atenção dos formadores, pois isso é crucial para criar um ambiente seguro e obter sucesso na prática. Isso nos leva à uma reflexão quanto aos 
critérios para a escolha do $\operatorname{coach}^{66}$. Se o aluno/cliente, não conhece da modalidade, pouco pode cobrar resultados da mesma e de quem oferece. Uma pesquisa com 193 instrutores Crossfit nos Estados Unidos da América mostrou que: $86 \%$ relataram ser um instrutor Crossfit certificado; $27 \%$ possuíam diploma de bacharel em um campo relacionado ao exercício; os instrutores com certificação Crossfit têm menos diplomas de bacharelado ou mestrado quando comparados aos que não possuem certificação Crossfit. Os instrutores relatam diferenças em sua educação e diferenças no uso de plataformas de levantamento de peso e vários tipos de calçados, ao ensino do levantamento de peso olímpico e na programação de exercícios ${ }^{7}$.

Este dado é extremamente relevante uma vez que no Brasil exige-se que todos os instrutores tenham, além da certificação em Crossfit, graduação em Educação Física. O que pode garantir maior preparo e segurança para a aplicação da prática, visto que o Crossfit falha em alguns princípios do treinamento físico como mencionado anteriormente. A sugestão aqui dada é que praticantes da modalidade cobrem de seu coach as devidas certificações e informações necessárias para guiá-los no processo seguro de prática física.

Por fim, a maioria dos indivíduos que responderam o questionário indicariam sem nenhuma dúvida o treinamento de Crossfit para outra pessoa. Isso pode ser relacionado com o fato de os entrevistados perceberem o ambiente do box do Crossfit motivador e acolhedor. Devemos levar em conta os cautelosos $4 \%$ que percebem na prática algum risco para aqueles que não possuem aptidão ou condições físicas para o Crossfit.

CONCLUSÃO

Este trabalho buscou analisar o grau de conhecimento e percepções dos praticantes do método Crossfit. Diante dos resultados é possível dizer que tal conhecimento não seja tão exato. Uma vez que eles buscam no treinamento desta modalidade saúde, competição, lazer, relacionamento social e estético onde todos realizam o mesmo treino no box. Os entrevistados e praticantes da metodologia responderam a respeito do seu próprio conhecimento ao método Crossfit, e a grande maioria diz ter conhecimento razoável ou pouco. Percebe-se com clareza tal fato, quando questionados a respeito do objetivo da sua prática esportiva selecionaram duas ou três alternativas, totalmente dicotômicas conceitualmente. Pelo fato de o Crossfit ser caracterizado um treinamento em alta intensidade, deve-se tomar cuidado com sua prescrição coletiva.

Assim, sugere-se aos coaches que os treinos sejam classificados de maneira diferente de acordo com os objetivos listados pelos alunos, para que assim possam obter resultados específicos. Ficou nítida, a importância do papel do coach no seu box, servindo como referência aos seus alunos, desde a respeito da parte técnica, até sua própria personalidade, bem como observável em qualquer outra prática de atividade física. Por fim é importante observar que, ainda falta aprofundamento técnico por parte dos alunos em relação ao método Crossfit, não deixando à mercê de condições puramente mercadológicas sua prática. Ficou evidente ainda que, mais estudos sobre o assunto são necessários para o melhor entendimento do treinamento desta metodologia.

REFERÊNCIAS

1. Glassaman G. What Is Fitness? CrossFit J 2002;1-11.

2. Glassaman G. What Is CrossFit? CrossFit J 2004;19:1-7.

3. Tibana A, Almeida LM, Prestes J. CrossFit $\AA$ riscos ou benefícios? O que sabemos até o momento? Rev Bras Ciência e Mov 2015; 23:182-85.

4. Costa D. Perspectivas atuais sobre o CrossFit. Grup. Estud. Avançados em Saúde e Exerc. GEASE. 2014.

5. Sabino JC, Cavalcante JF, Neto LTR, Neto VAS, Melo EL, Félicio LF et al. CrossFit e musculação: aspectos do condicionamento físico, psicológico e motivacional. Coleção Pesqui em Educ Física 2016;11:59-68.

6. Heinrich KM, Carlisle T, Kehler A, Cosgrove SJ. Mapping Coaches' Views of Participation in CrossFit to the Integrated Theory of Health Behavior Change and Sense of Community. Fam Community Health 2017;40:24-7.

7. Waryasz GR, Suric $V$, Daniels AH, Gil JA, Eberson CP. CrossFit $\AA$ Instructor Demographics and Practice Trends. Orthop Rev (Pavia) 2016;8:65-71.

8. E scobar KA, Morales J, Vandusseldorp TA. The Effect of a Moderately Low and High Carbohydrate Intake on Crossfit Performance. Int J Exerc Sci 2016; 9:460-70.

9. K ramer SJ, Baur DA, Spicer MT, Vukovich MD, Ormsbee MJ. The effect of six days of dietary nitrate supplementation on performance in trained CrossFit athletes. J Int Soc Sports Nutr 2016;13:39.

10. Outlaw JJ, Wilborn CD, Smith-Ryan AE, Hayward SE, Urbina SL, Taylor LW et al. Effects of a pre-and post-workout proteincarbohydrate supplement in trained crossfit 
individuals. Springerplus 2014;3:369.

11. Fisher J, Sales A, Carlson L, Steele J. A comparison of the motivational factors between CrossFit participants and other resistance exercise modalities: a pilot study. J Sport Med Phys Fit. 2017;57:1227-34.

12. Partridge JA, Knapp BA, Massengale BD. An Investigation of Motivational Variables in CrossFit Facilities. J Strength Cond Res. 2014; 28: 1714-21.

13. Whiteman-Sandland J, Hawkins J, Clayton D. The role of social capital and community belongingness for exercise adherence: An exploratory study of the CrossFit gym model. J Health Psychol. 2018;23(12):1545-56.

14. Tafuri S, Notarnicola A, Monno A, Ferretti F, Moretti B. CrossFit athletes exhibit high symmetry of fundamental movement patterns. A cross-sectional study. Muscles Ligaments Tendons J. 2016;6(1):157-60.

15. Montalvo AM, Shaefer $H$, Rodriguez B, Li T, Epnere K, Myer GD. Retrospective Injury Epidemiology and Risk Factors for Injury in CrossFit. J Sports Sci Med. 2017;16(1):53-9.

16. Moran S, Booker H, Staines J, Williams S. Rates and risk factors of injury in CrossFitTM: a prospective cohort study. J Sports Med Phys Fitness. 2017;57(9):1147-53.

17. Hak PT, Hodzovic E, Hickey B. The nature and prevalence of injury during CrossFit training. $\mathrm{J}$ Strength Cond Res. 2013. doi: 10.1519/JSC. 0000000000000318 .

18. Summitt RJ, Cotton RA, Kays AC, Slaven EJ. Shoulder Injuries in Individuals Who Participate in CrossFit Training. Sports Health. 2016; 8(6):541-46.

19. Sprey JW, Ferreira T, de Lima MV, Duarte A Jr, Jorge PB, Santili C. An Epidemiological Profile of CrossFit Athletes in Brazil. Orthop J Sports Med. 2016;4(8):2325967116663706.

20. Mehrab M, de Vos RJ, Kraan GA, Mathijssen NMC. Injury Incidence and Patterns Among Dutch CrossFit Athletes. Orthop J Sports Med. 2017;5(12):2325967117745263.

21. Fisker FY, Kildegaard S, Thygesen M, Grosen $\mathrm{K}$, Pfeiffer-Jensen M. Acute tendon changes in intense CrossFit workout: an observational cohort study. Scand J Med Sci Sports. 2017;27(11):1258-62.

22. Friedman MV, Stensby JD, Hillen TJ, Demertzis JL, Keener JD. Traumatic tear of the latissimus dorsi myotendinous junction: case report of a crossfit-related injury. Sports Health. 2015;7(6):548-52.

23. Cunha GV, Prestes J, Voltarelli FAR, Tibana A. Rabdomiólise em programas de condicionamento extremo. Rev Bras Fisiol do Exerc 2017;16(4):234-40.

24. Larsen C, Jensen MP. [Rhabdomyolysis in a well-trained woman after unusually intense exercise]. Ugeskr Laeger. 2014.;176(25):V01140001.

25. Hadeed MJ, Kuehl KS, Elliot DL, Sleigh A. Exertional Rhabdomyolysis After Crossfit Exercise Program. Med. Sci Sports Exerc. 2011;43:224-25.

26. Aynardi MC, Jones CM. Bilateral upper arm compartment syndrome after a vigorous crosstraining workout. J Shoulder Elbow Surg. 2016;25(3):e65-7.

27. Lozowska D, Liewluck T, Quan D, Ringel SP. Exertional rhabdomyolysis associated with high intensity exercise. Muscle Nerve. 2015;52(6):1134-35.

28. Meyer M, Sundaram S, Schafhalter-Zoppoth I. Exertional and CrossFit-Induced Rhabdomyolysis. Clin J Sport Med. 2018; 28(6):e92-4.

29. Maté-Muñoz JL, Lougedo JH, Barba M, GarcíaFernández P, Garnacho-Castaño MV, Domínguez $R$. Muscular fatigue in response to different modalities of CrossFit sessions. PLoS One. 2017;12(7):e0181855.

30. Middlekauff ML, Egger MJ, Nygaard IE, Shaw JM. The impact of acute and chronic strenuous exercise on pelvic floor muscle strength and support in nulliparous healthy women. Am J Obstet Gynecol. 2016;215(3):316.e1-7.

31. Murawska-Cialowicz E, Wojna J, ZuwalaJagiello J. Crossfit training changes brainderived neurotrophic factor and irisin levels at rest, after wingate and progressive tests, and improves aerobic capacity and body composition of young physically active men and women. J Physiol Pharmacol. 2015; 66(6):811-21.

32. Ouellette KA, Brusseau TA, Davidson LE, Ford CN, Hatfield DL, Shaw JM, Eisenman PA. Comparison of the Effects of Seated, Supine, and Walking Interset Rest Strategies on Work Rate. J Strength Cond Res. 2016;30(12):3396404.

33. Bellar D, Hatchett A, Judge LW, Breaux ME, Marcus $L$. The relationship of aerobic capacity, anaerobic peak power and experience to performance in CrossFit exercise. Biol Sport. 2015;32(4):315-20.

34. Tibana RA, de Almeida LM, Frade de Sousa NM, Nascimento Dda C, Neto IV, de Almeida JA et al. Two Consecutive Days of Crossfit Training Affects Pro and Anti-inflammatory Cytokines and Osteoprotegerin without Impairments in Muscle Power. Front Physiol. 2016;7:260.

35. Serafini PR, Feito Y, Mangine GT. Self-reported Measures of Strength and Sport-Specific Skills Distinguish Ranking in an International Online Fitness Competition. J Strength Cond Res. 2018;32(12):3474-84.

36. Ward JK, Hastie PA, Wadsworth DD, Foote S, 
Brock SJ, Hollett N. A Sport Education Fitness Season's Impact on Students' Fitness Levels, Knowledge, and In-Class Physical Activity. Res Q Exerc Sport. 2017;88(3):346-51.

37. Poston WS, Haddock CK, Heinrich KM, Jahnke SA, Jitnarin N, Batchelor DB. Is High-Intensity Functional Training (HIFT)/CrossFit Safe for Military Fitness Training? Mil Med. 2016;181(7):627-37.

38. Grier T, Canham-Chervak M, McNulty V, Jones $\mathrm{BH}$. Extreme conditioning programs and injury risk in a US Army Brigade Combat Team. US Army Med Dep J. 2013:36-47.

39. O'Connor FG, Deuster PA, Barrett J, Kane SF, Depenbrock P. Letter: Is High-Intensity Functional Training (HIFT)/CrossFit Safe for Military Fitness Training? Mil Med. 2017;182(1):1474-75.

40. Haddock CK, Poston WS, Heinrich KM, Jahnke SA, Jitnarin N. The Benefits of High-Intensity Functional Training Fitness Programs for Military Personnel. Mil Med. 2016;181(11):e1508-514.

41. Eather N, Morgan PJ, Lubans DR. Improving health-related fitness in adolescents: the CrossFit Teens ${ }^{\mathrm{TM}}$ randomised controlled trial. J Sports Sci. 2016;34(3):209-23.

42. Claudino JG, Gabbett TJ, Bourgeois F, Souza HS, Miranda RC, Mezêncio $B$ et al. CrossFit Overview: Systematic Review and Metaanalysis. Sports Med Open. 2018;4(1):11.

43. Tubino MJG. Dimensões sociais do esporte. Cortez: São Paulo; 2001.

44. Lakatos EM, Marconi MA. Fundamentos da Metodologia Científica. 5. ed. Atlas: São Paulo; 2003.

45. Thomas JR, Nelson JK, Silverman SJ. Métodos de pesquisa em atividade física. 6t. ed. Artmed: Porto Alegre; 2012.

46. Anzai K. O corpo enquanto objeto de consumo. RBCE. 2000;21(2):71-6.

47. Sant'Anna DB. Identidade Corporal. In: Corpo, Prazer e Movimento. SESC: São Paulo, 2002.

48. Marcellino NCNC. Academias de ginástica como opção de lazer. R Bras $\mathrm{Ci}$ e Mov 2003;11(2):49-54.

49. Dumazedier J. Sociologia empírica do lazer. Perspectiva: São Paulo; 1979.

50. Machado CV. Motivos da procura e desistência da prática de atividade física em uma academia de Santa Rosa-RS [monografia]. ljuí: Universidade Regional do Noroeste do Estado do RS - UNIJUÍ; 2016.

51. Bircher J. Towards a dynamic definition of health and disease. Med Health Care Philos. 2005;8(3):335-41.

52. World Health Organization. Preamble to the Constitution of the World Health Organization as adopted by the International Health Conference. In: International Health
Conference. New York, 1948.

53. CrossFit Inc. Guia de Treinamento de nível 1. CrossFit. 2018. Disponível em: https://autoridadecross.com.br/wp-content/ uploads/2019/06/GUIA-DE-TREINAMENTODE-N\%C3\%8DVEL-1.pdf.

54. Knapik JJ. Extreme Conditioning Programs: Potential Benefits and Potential Risks. J Spec Oper Med. 2015;15(3):108-13.

55. Klimek C, Ashbeck C, Brook AJ, Durall C. Are Injuries More Common With CrossFit Training Than Other Forms of Exercise? J Sport Rehabil. 2018;27(3):295-99.

56. Tubino MJG. Metodologia científica do treinamento desportivo. 3. ed. Ibrasa: São Paulo, 1984.

57. Weineck J. Biologia do Esporte. Manole: São Paulo; 1991.

58. Dantas EHM. A prática da preparação física. 3. ed. Shape: Rio de Janeiro; 1995.

59. Xavier A, Lopes AM. Lesões Musculoesqueléticas em Praticantes de CrossFit. RICM 2017;1(1):11-27.

60. Nóbrega $A C L$, Freitas EV, Oliveira MAB, Leitão MB, Lazzoli JK, Nahas RM et al . Posicionamento oficial da Sociedade Brasileira de Medicina do Esporte e da Sociedade Brasileira de Geriatria e Gerontologia: atividade física e saúde no idoso. Rev Bras Med Esporte. 1999;5(6):207-11.

61. Fleck MPA. O instrumento de avaliação de qualidade de vida da Organização Mundial da Saúde (WHOQOL-100): características e perspectivas. Ciênc saude coletiva. 2000;5(1):33-8.

62. The World Health Organization Quality of Life assessment (WHOQOL): position paper from the World Health Organization. Soc Sci Med. 1995;41(10):1403-9.

63. Souza DC, Arruda A, Gentil P. CROSSFIT®: riscos para possíveis benefícios? RBPFEx. 2017; 11(64):138-39.

64. Bosch X, Poch E, Grau JM. Rhabdomyolysis and acute kidney injury. N Engl J Med. 2009;361(1):62-72.

65. CrossFit Inc. How to start CrossFit? CossFit; 2017.

66. Weisenthal BM, Beck CA, Maloney MD, DeHaven KE, Giordano BD. Injury Rate and Patterns Among CrossFit Athletes. Orthop J Sports Med. 2014;2(4):2325967114531177. 


\section{CONFLITO DE INTERESSES}

Os autores declaram não haver conflitos de interesse

\section{AUTOR PARA CORRESPONDÊNCIA}

\section{Daniel Zonzini Voltan}

Centro Universitário de Jaguariúna - UNIFAJ

Rua Nelson Righi, 47, Ap. 34, Parque dos Ipês, 13912-

428 Jaguariúna - SP, Brasil

e-mail: danielzvoltan@gmail.com

Submetido em 20/12/2019

Aceito em 22/10/2020 